\title{
Lorentz-Schatten classes of direct sum of operators
}

\author{
Pembe Ipek $\mathrm{Al}$ \\ Karadeniz Technical University, Department of Mathematics, 61080, Trabzon, Turkey
}

\begin{abstract}
In this paper, the relations between Lorentz-Schatten property of the direct sum of operators and Lorentz-Schatten property of its coordinate operators are studied. Then, the results are supported by applications.
\end{abstract}

Mathematics Subject Classification (2010). 47A05, 47A10

Keywords. direct sum of Hilbert spaces and operators, compact operators, Lorentz-Schatten operator classes

\section{Introduction}

The general theory of singular numbers and operator ideals was given by Pietsch $[13,14]$ and the case of linear compact operators was investigated by Gohberg and Krein [5]. However, the first result in this area can be found in the works of Schmidt [16] and Schatten, von Neumann [15]. They used these concepts in the theory of non-selfadjoint integral equations.

Later on, the main aim of mini-workshop held in Oberwolfach (Germany) was to present and discuss some modern applications of the functional-analytic concepts of $s$-numbers and operator ideals in areas like numerical analysis, theory of function spaces, signal processing, approximation theory, probability of Banach spaces and statistical learning theory (see [3]).

Let $\mathcal{H}$ be a Hilbert space, $S_{\infty}(\mathcal{H})$ be a class of linear compact operators in $\mathcal{H}$ and $s_{n}(T)$ be the $n-t h$ singular numbers of the operator $T \in S_{\infty}(\mathcal{H})$. The Lorentz-Schatten operator ideals are defined as

$$
S_{p, q}(\mathcal{H})=\left\{T \in S_{\infty}(\mathcal{H}): \sum_{n=1}^{\infty} n^{\frac{q}{p}-1} s_{n}^{q}(T)<\infty\right\}, 0<p \leq \infty, 0<q<\infty
$$

and

$$
S_{p, \infty}(\mathcal{H})=\left\{T \in S_{\infty}(\mathcal{H}): \sup _{n \geq 1} n^{\frac{1}{p}} s_{n}(T)<\infty\right\}, 0<p \leq \infty
$$

in $[1,13,14,17]$.

Let $\alpha$ be a positive real number. If $s_{n}(T) \sim c n^{-\alpha}, c>0, n \rightarrow \infty$ for any linear compact operator $T$ in a Hilbert space $\mathcal{H}$, then for each $p \in\left(\frac{1}{\alpha}, \infty\right]$ and $q \in(0, \infty), T \in S_{p, q}(\mathcal{H})$. In

Email address: ipekpembe@gmail.com

Received: 05.02.2019; Accepted: 10.04.2019 
this case, the necessary and sufficient condition for the series $\sum_{n=1}^{\infty} n^{\frac{q}{p}-1-\alpha q}$ to be convergent is $p>\frac{1}{\alpha}$. Moreover, the necessary and sufficient condition for $T \in S_{p, \infty}(\mathcal{H})$ is $p \in\left[\frac{1}{\alpha}, \infty\right]$.

The infinite direct sum of Hilbert spaces and the infinite direct sum of operators have been studied in [4]. Namely, the infinite direct sum of Hilbert spaces $H_{n}, n \geq 1$ and the infinite direct sum of operators $A_{n}$ in $H_{n}, n \geq 1$ are defined as

$$
\begin{gathered}
H=\bigoplus_{n=1}^{\infty} H_{n}=\left\{u=\left(u_{n}\right): u_{n} \in H_{n}, n \geq 1, \sum_{n=1}^{\infty}\left\|u_{n}\right\|_{H_{n}}^{2}<+\infty\right\}, \\
A=\bigoplus_{n=1}^{\infty} A_{n}, \\
D(A)=\left\{u=\left(u_{n}\right) \in H: u_{n} \in D\left(A_{n}\right), n \geq 1, A u=\left(A_{n} u_{n}\right) \in H\right\} .
\end{gathered}
$$

Recall that $H$ is a Hilbert space with the norm induced by the inner product

$$
(u, v)_{H}=\sum_{n=1}^{\infty}\left(u_{n}, v_{n}\right)_{H_{n}}, u, v \in H .
$$

Our aim in this paper is to study the relations between Lorentz-Schatten property of the direct sum of operators and Lorentz-Schatten property of its coordinate operators.

It should be noted that the analogous problems in special cases have been investigated in $[8]$.

The problem of belonging to the Schatten-von Neuman classes of the resolvent operators of the normal extensions of the minimal operator generated by the direct sum of differential-operator expression for first order with suitable operator coefficients in the direct sum of Hilbert spaces in finite interval has been studied in [7].

In $[6,9]$, the same problem for normal and hyponormal extensions of the minimal operators generated by corresponding differential-operator expressions under some conditions to operator coefficients in a finite interval has been investigated.

Later on, some more general Schatten-von Neumann classes of compact operators in Hilbert spaces have been defined and characterized in [10] in terms of Berezin symbols. In [2], the question raised by Nordgren and Rosenthal about the Schatten-von Neumann class membership of operators in standard reproducing kernel Hilbert spaces in terms of their Berezin symbols has been answered.

\section{Lorentz-Schatten property of block diagonal operator matrices}

Let $H_{n}$ be a Hilbert space, $A_{n} \in L\left(H_{n}\right)$ for $n \geq 1$ and

$$
H=\bigoplus_{n=1}^{\infty} H_{n}, A=\bigoplus_{n=1}^{\infty} A_{n}
$$

Recall that, in order to $A \in L(H)$ the necessary and sufficient condition is $\sup _{n \geq 1}\left\|A_{n}\right\|<\infty$. Moreover, $\|A\|=\sup _{n \geq 1}\left\|A_{n}\right\|$ (see [11]).

It is known that if $A_{n} \in S_{\infty}\left(H_{n}\right)$ for $n \geq 1$, then the necessary and sufficient condition for $A \in S_{\infty}(H)$ is $\lim _{n \rightarrow \infty}\left\|A_{n}\right\|=0$ (see [12]).

The following result on singular numbers of the operator $A \in S_{\infty}(H)$

$$
\left\{s_{m}(A): m \geq 1\right\}=\bigcup_{n=1}^{\infty}\left\{s_{m}\left(A_{n}\right): m \geq 1\right\}
$$

can be found in [8].

Throughout this paper, for the simplicity we assume that: 
(1) for any $n, k \geq 1$ with $n \neq k,\left\{s_{m}\left(A_{n}\right): m \geq 1\right\} \cap\left\{s_{m}\left(A_{k}\right): m \geq 1\right\}=\emptyset$ or $\{0\}$;

(2) for any $n \geq 1$ in the sequence $\left(s_{m}\left(A_{n}\right)\right)$, if for some $k>1, s_{k}\left(A_{n}\right)>0$, then $s_{k}\left(A_{n}\right)<$ $s_{k-1}\left(A_{n}\right)$.

Proposition 2.1. For $n \geq 1$ there is a strongly increasing sequence $k_{m}^{(n)}: \mathbb{N} \rightarrow \mathbb{N}$ such that $s_{k_{m}^{(n)}}(A)=s_{m}\left(A_{n}\right)$ holds for $m \geq 1$ and $\bigcup_{n=1}^{\infty} \bigcup_{m=1}^{\infty}\left\{k_{m}^{(n)}\right\}=\mathbb{N}$. Moreover, it is clear that $k_{m}^{(n)} \geq m$ for $n, m \geq 1$.

Indeed, in the Hilbert space $H=\bigoplus_{n=1}^{\infty} H_{n}=l_{2}(\mathbb{R})$, where $H_{n}=(\mathbb{R},|\cdot|)$, consider the following infinite matrices with reel entries in forms

$$
A=\left(\begin{array}{cccccc}
a_{1} & & & & & \\
& a_{2} & & & & \\
& & a_{3} & & & 0 \\
& & & \ddots & & \\
& 0 & & & a_{n} & \\
& & & & & \ddots
\end{array}\right): H \rightarrow H
$$

and

$$
B=\left(\begin{array}{cccccc}
b_{1} & & & & & \\
& b_{2} & & & & \\
& & b_{3} & & & 0 \\
& & & \ddots & & \\
& 0 & & & b_{n} & \\
& & & & & \ddots
\end{array}\right): H \rightarrow H,
$$

where for any $n, m \geq 1, n \neq m, a_{n} \neq a_{m}, a_{n}>0$ and $b_{n}=\frac{a_{n}+a_{n+1}}{2}$ with property $\lim _{n \rightarrow \infty} a_{n}=0$.

In this case, $A, B \in S_{\infty}(H)$ and the singular numbers of the operators $A, B$ are given in the following forms

$$
\begin{aligned}
& \left\{s_{m}\left(A_{n}\right): m \geq 1\right\}=\left\{a_{n}: n \geq 1\right\}, \\
& \left\{s_{m}\left(B_{n}\right): m \geq 1\right\}=\left\{b_{n}: n \geq 1\right\},
\end{aligned}
$$

respectively. Then, by [12] it implies that $T=A \oplus B \in S_{\infty}(H \oplus H)$ and $\left\{s_{m}(T): m \geq 1\right\}=$ $\left\{a_{n}, b_{n}: n \geq 1\right\}$. In this case, it is easy to see that

$$
\begin{aligned}
& k_{m}^{(1)}=2 m-1, m \geq 1, \\
& k_{m}^{(2)}=2 m, m \geq 1 .
\end{aligned}
$$

Theorem 2.2. Let $0<p, q<\infty . A \in S_{p, q}(H)$ if and only if the series

$$
\sum_{n=1}^{\infty} \sum_{m=1}^{\infty}\left(k_{m}^{(n)}\right)^{\frac{q}{p}-1} s_{m}^{q}\left(A_{n}\right)
$$

is convergent.

Proof. If $A \in S_{p, q}(H)$, it is clear that the series

$$
\sum_{m=1}^{\infty} m^{\frac{q}{p}-1} s_{m}^{q}(A)
$$


is convergent. From the structure of the set of the singular numbers of the operator $A$ and the important theorem on the convergent of the rearrangement series it is obtained that the series

is convergent.

$$
\sum_{n=1}^{\infty} \sum_{m=1}^{\infty}\left(k_{m}^{(n)}\right)^{\frac{q}{p}-1} s_{m}^{q}\left(A_{n}\right)
$$

Conversely, if the series in the theorem is convergent, then $\sum_{m=1}^{\infty} m^{\frac{q}{p}-1} s_{m}^{q}(A)$, which is the rearrangement of the above series, is convergent. So, $A \in S_{p, q}(H)$.

Now, in Theorem 2.3-2.5, we will investigate the problem of belonging to LorentzSchatten classes of its coordinate operators, if the direct sum of operators belongs to Lorentz-Schatten classes.

Theorem 2.3. Let $A \in S_{\infty}(H)$ and $0<p \leq q<\infty$. If $A \in S_{p, q}(H)$, then $A_{n} \in S_{p, q}\left(H_{n}\right)$ for $n \geq 1$.

Proof. In the special case $0<p=q<\infty$, the result has been proved in [8].

In the case of $p<q$, we have

$$
m \leq k_{m}^{(n)} \text { and } s_{k_{m}^{(n)}}(A)=s_{m}\left(A_{n}\right)
$$

for $n, m \geq 1$. Consequently, for $n \geq 1$ we get

$$
\begin{aligned}
\sum_{m=1}^{\infty} m^{\frac{q}{p}-1} s_{m}^{q}\left(A_{n}\right) & \leq \sum_{m=1}^{\infty}\left(k_{m}^{(n)}\right)^{\frac{q}{p}-1} s_{m}^{q}\left(A_{n}\right) \\
& \leq \sum_{n=1}^{\infty} \sum_{m=1}^{\infty}\left(k_{m}^{(n)}\right)^{\frac{q}{p}-1} s_{m}^{q}\left(A_{n}\right) \\
& =\sum_{m=1}^{\infty} m^{\frac{q}{p}-1} s_{m}^{q}(A)<\infty .
\end{aligned}
$$

Hence, $A_{n} \in S_{p, q}\left(H_{n}\right)$ for $n \geq 1$.

Theorem 2.4. Let $0<q<p<\infty$ and for $n \geq 1$, $\sup _{m \geq 1}\left(\frac{k_{m}^{(n)}}{m}\right) \leq \gamma<\infty$. If $A \in S_{p, q}(H)$, then $A_{n} \in S_{p, q}\left(H_{n}\right)$ for $n \geq 1$.

Proof. Under the assumptions in the theorem, we have

$$
\begin{aligned}
\sum_{m=1}^{\infty} m^{\frac{q}{p}-1} s_{m}^{q}\left(A_{n}\right) & =\sum_{m=1}^{\infty}\left(\frac{m}{k_{m}^{(n)}}\right)^{\frac{q}{p}-1}\left(k_{m}^{(n)}\right)^{\frac{q}{p}-1} s_{m}^{q}\left(A_{n}\right) \\
& \leq \sup _{m \geq 1}\left(\frac{k_{m}^{(n)}}{m}\right)^{1-\frac{q}{p}} \sum_{m=1}^{\infty}\left(k_{m}^{(n)}\right)^{\frac{q}{p}-1} s_{m}^{q}\left(A_{n}\right) \\
& \leq \gamma^{1-\frac{q}{p}} \sum_{n=1}^{\infty} \sum_{m=1}^{\infty}\left(k_{m}^{(n)}\right)^{\frac{q}{p}-1} s_{m}^{q}\left(A_{n}\right) \\
& =\gamma^{1-\frac{q}{p}} \sum_{j=1}^{\infty} j^{\frac{q}{p}-1} s_{j}^{q}(A)<\infty .
\end{aligned}
$$

Therefore, $A_{n} \in S_{p, q}\left(H_{n}\right)$ for $n \geq 1$.

Now, we will investigate the case of $q=\infty$. 
Theorem 2.5. Let $0<p \leq \infty$. If $A \in S_{p, \infty}(H)$, then $A_{n} \in S_{p, \infty}\left(H_{n}\right)$ for $n \geq 1$.

Proof. Since $A \in S_{p, \infty}(H)$, we have $\sup _{m \geq 1} m^{\frac{1}{p}} s_{m}(A)<\infty$. Hence, $\sup _{m \geq 1}\left(k_{m}^{(n)}\right)^{\frac{1}{p}} s_{m}\left(A_{n}\right)<$ $\infty$. On the other hand, we get

$$
\begin{aligned}
\sup _{m \geq 1} m^{\frac{1}{p}} s_{m}\left(A_{n}\right) & =\sup _{m \geq 1}\left(k_{m}^{(n)}\right)^{\frac{1}{p}} s_{m}\left(A_{n}\right)\left(\frac{m}{k_{m}^{(n)}}\right)^{\frac{1}{p}} \\
& \leq \sup _{m \geq 1}\left(k_{m}^{(n)}\right)^{\frac{1}{p}} s_{m}\left(A_{n}\right)<\infty .
\end{aligned}
$$

Then, $A_{n} \in S_{p, \infty}\left(H_{n}\right)$ for $n \geq 1$.

Now, in Theorem 2.6-2.8, we will investigate the problem of belonging to LorentzSchatten classes of the direct sum of operators, if its coordinate operators belong to Lorentz-Schatten classes.

Theorem 2.6. Let $0<q \leq p<\infty$. If $A_{n} \in S_{p, q}\left(H_{n}\right)$ for $n \geq 1$ and the series $\sum_{n=1}^{\infty} \sum_{m=1}^{\infty} m^{\frac{q}{p}-1} s_{m}^{q}\left(A_{n}\right)$ is convergent, then $A \in S_{p, q}(H)$.

Proof. For $0<q \leq p<\infty$, we have

$$
\begin{aligned}
\sum_{m=1}^{\infty} m^{\frac{q}{p}-1} s_{m}^{q}(A) & =\sum_{n=1}^{\infty} \sum_{m=1}^{\infty}\left(k_{m}^{(n)}\right)^{\frac{q}{p}-1} s_{k_{m}^{q}(n)}(A) \\
& =\sum_{n=1}^{\infty} \sum_{m=1}^{\infty}\left(\frac{k_{m}^{(n)}}{m}\right)^{\frac{q}{p}-1} m^{\frac{q}{p}-1} s_{m}^{q}\left(A_{n}\right) \\
& \leq \sum_{n=1}^{\infty} \sum_{m=1}^{\infty} m^{\frac{q}{p}-1} s_{m}^{q}\left(A_{n}\right)<\infty .
\end{aligned}
$$

This completes the proof.

Theorem 2.7. Let $0<p<q<\infty$, for $n \geq 1 \sum_{m=1}^{\infty} m^{\frac{q}{p}-1} s_{m}^{q}\left(A_{n}\right) \leq \beta_{n}<\infty$, $\sup _{m \geq 1}\left(\frac{k_{m}^{(n)}}{m}\right) \leq$ $\gamma_{n}<\infty$ and $\sum_{n=1}^{\infty} \gamma_{n}^{\frac{q}{p}-1} \beta_{n}<\infty$. If $A_{n} \in S_{p, q}\left(H_{n}\right)$ for $n \geq 1$, then $A \in S_{p, q}(H)$.

Proof. The validity of this claim is clear from the following inequality

$$
\begin{aligned}
\sum_{m=1}^{\infty} m^{\frac{q}{p}-1} s_{m}^{q}(A) & =\sum_{n=1}^{\infty} \sum_{m=1}^{\infty}\left(k_{m}^{(n)}\right)^{\frac{q}{p}-1} s_{k_{m}^{q}(n)}^{q}(A) \\
& =\sum_{n=1}^{\infty} \sum_{m=1}^{\infty}\left(\frac{k_{m}^{(n)}}{m}\right)^{\frac{q}{p}-1} m^{\frac{q}{p}-1} s_{m}^{q}\left(A_{n}\right) \\
& \leq \sum_{n=1}^{\infty}\left(\sup _{m \geq 1}\left(\frac{k_{m}^{(n)}}{m}\right)\right)^{\frac{q}{p}-1} \sum_{m=1}^{\infty} m^{\frac{q}{p}-1} s_{m}^{q}\left(A_{n}\right) \\
& \leq \sum_{n=1}^{\infty} \gamma_{n}^{\frac{q}{p}-1} \beta_{n} .
\end{aligned}
$$

Now, we will investigate in the case of $q=\infty$. 
Theorem 2.8. Let $0<p<\infty$, for $n \geq 1 \alpha_{n}=\sup _{m \geq 1}\left(\frac{k_{m}^{(n)}}{m}\right)^{\frac{1}{p}}<\infty, \gamma_{n}=\sup _{m \geq 1} m^{\frac{1}{p}} s_{m}\left(A_{n}\right)$ and $\sup _{n \geq 1} \alpha_{n} \gamma_{n}<\infty$. If $A_{n} \in S_{p, \infty}\left(H_{n}\right)$ for $n \geq 1$, then $A \in S_{p, \infty}(H)$.

Proof. This result is clear from the following relation

$$
\begin{aligned}
\sup _{m \geq 1} m^{\frac{1}{p}} s_{m}(A) & =\sup _{n, m \geq 1}\left(k_{m}^{(n)}\right)^{\frac{1}{p}} s_{k_{m}^{(n)}}(A) \\
& =\sup _{n, m \geq 1}\left(k_{m}^{(n)}\right)^{\frac{1}{p}} s_{m}\left(A_{n}\right) \\
& \leq \sup _{n \geq 1}\left(\sup _{m \geq 1}\left(\frac{k_{m}^{(n)}}{m}\right)^{\frac{1}{p}} \sup _{m \geq 1} m^{\frac{1}{p}} s_{m}\left(A_{n}\right)\right) \\
& =\sup _{n \geq 1} \alpha_{n} \gamma_{n}<\infty .
\end{aligned}
$$

Theorem 2.9. Let $0<p_{n}, q_{n}<\infty, A_{n} \in S_{p_{n}, q_{n}}\left(H_{n}\right)$ for $n \geq 1$ and $p=\sup _{n \geq 1} p_{n}<\infty, q=$ $\sup _{n \geq 1} q_{n}<\infty$. Then, $A \in S_{p, q}(H)$ if and only if the series $\sum_{n=1}^{\infty} \sum_{m=1}^{\infty}\left(k_{m}^{(n)}\right)^{\frac{q}{p}-1} s_{m}^{q}\left(A_{n}\right)$ is convergent.

Proof. From the result in [1], we have $A_{n} \in S_{p, q}\left(H_{n}\right)$ for $n \geq 1$. Therefore, the validity of this claim is implied by Theorem 2.2 .

Remark 2.10. Using this method, the analogous researches for the following operators

$$
B=\left(\begin{array}{ccccccc}
0 & B_{1} & & & & & \\
& 0 & B_{2} & & & & \\
& & 0 & B_{3} & & 0 & \\
& & & \ddots & \ddots & & \\
& 0 & & & 0 & B_{n} & \\
& & & & & \ddots & \ddots
\end{array}\right): H=\bigoplus_{n=1}^{\infty} H_{n} \rightarrow H
$$

and

$$
C=\left(\begin{array}{cccccc}
0 & & & & & \\
C_{1} & 0 & & & & \\
& C_{2} & 0 & & & 0 \\
& & \ddots & \ddots & & \\
& 0 & & C_{n} & 0 & \\
& & & & \ddots & \ddots
\end{array}\right): H=\bigoplus_{n=1}^{\infty} H_{n} \rightarrow H
$$

can be studied. 


\section{Examples}

In this section, we provide some examples as applications of our theorems.

Example 3.1. In the Hilbert space $H=\bigoplus_{n=1}^{\infty} H_{n}=l_{2}(\mathbb{C})$, where $H_{n}:=(\mathbb{C},|\cdot|), n \geq 1$, consider the following diagonal infinite matrix with complex entries

$$
A=\left(\begin{array}{cccccc}
a_{1} & & & & & \\
& a_{2} & & & & \\
& & a_{3} & & & 0 \\
& & & \ddots & & \\
& 0 & & & a_{n} & \\
& & & & & \ddots
\end{array}\right): H \rightarrow H
$$

under the condition $\left|a_{n}\right|<r<1, n \geq 1$. Then, $\lim _{n \rightarrow \infty} a_{n}=0$. In this case, $A \in S_{\infty}(H)$. If we define $A_{n}:=a_{n}$ for $n \geq 1$, then $s_{m}\left(A_{n}\right)=\left|\lambda\left(A_{n}\right)\right|=\left\{\left|a_{n}\right|, 0\right\}, m \geq 1$.

Hence, the singular numbers of the operator $A$ are given as

$$
\left\{s_{m}(A): m \geq 1\right\}=\left\{\left|a_{n}\right|: n \geq 1\right\} .
$$

On the other hand, for $n \geq 1$ and $0<q \leq p<\infty$ we get

$$
\sum_{m=1}^{\infty} m^{\frac{q}{p}-1} s_{m}^{q}\left(A_{n}\right)=\left|a_{n}\right|^{q}
$$

Then, $A_{n} \in S_{p, q}\left(H_{n}\right), n \geq 1,0<q \leq p<\infty$. Therefore, we have

$$
\sum_{n=1}^{\infty} \sum_{m=1}^{\infty} m^{\frac{q}{p}-1} s_{m}^{q}\left(A_{n}\right)=\sum_{n=1}^{\infty}\left|a_{n}\right|^{q}<\infty
$$

Hence, by Theorem 2.6, $A \in S_{p, q}(H)$.

Example 3.2. Let $H_{n}:=\left(\mathbb{C}^{2},|\cdot|_{2}\right), H:=\bigoplus_{n=1}^{\infty} H_{n}=l_{2}\left(\mathbb{C}^{2}\right), A_{n}=\left(\begin{array}{cc}0 & \alpha^{2 n-1} \\ \alpha^{2 n} & 0\end{array}\right)$ for $n \geq 1,0<|\alpha|<1$ and $A=\bigoplus_{n=1}^{\infty} A_{n}: H \rightarrow H$. Then $A \in S_{\infty}(H)$ (see [12]).

In this case, for $n \geq 1$ we get

and

$$
\begin{gathered}
\left\|A_{n}\right\|=|\alpha|^{2 n-1}, \\
\left\{s_{m}\left(A_{n}\right): m \geq 1\right\}=\left\{|\alpha|^{2 n-1},|\alpha|^{2 n}\right\}
\end{gathered}
$$

$$
\left\{s_{m}(A): m \geq 1\right\}=\left\{|\alpha|^{n}: n \geq 1\right\} .
$$

On the other hand, for $n \geq 1$ and $0<q \leq p<\infty$ we obtain

$$
\sum_{m=1}^{\infty} m^{\frac{q}{p}-1} s_{m}^{q}\left(A_{n}\right)=|\alpha|^{(2 n-1) q}+2^{\frac{q}{p}-1}|\alpha|^{2 n q}<\infty .
$$

Hence, $A_{n} \in S_{p, q}\left(H_{n}\right), n \geq 1,0<q \leq p<\infty$. Therefore, we have

$$
\sum_{n=1}^{\infty} \sum_{m=1}^{\infty} m^{\frac{q}{p}-1} s_{m}^{q}\left(A_{n}\right)=\sum_{n=1}^{\infty}\left(|\alpha|^{(2 n-1) q}+2^{\frac{q}{p}-1}|\alpha|^{2 n q}\right)=\frac{|\alpha|^{q}}{1-|\alpha|^{2 q}}\left(1+2^{\frac{q}{p}-1}|\alpha|^{q}\right)<\infty .
$$

Hence, by Theorem 2.6, $A \in S_{p, q}(H)$. 
Acknowledgment. The author would like to thank Professor Z. I. Ismailov (Karadeniz Technical University, Department of Mathematics, Turkey) for his various comments and suggestions.

\section{References}

[1] M.Sh. Birman and M.Z. Solomyak, Estimates of singular numbers of integral operators, Russian Math. Survey 32 (1), 15-89, 1977 (Translated from Uspekhi Mat. Nauk 32 (1), 17-84, 1977).

[2] I. Chalendar, E. Fricain, M. Gürdal and M. T. Karaev, Compactness and Berezin symbols, Acta Sci. Math. (Szeged) 78 (1), 315-329, 2012.

[3] F. Cobos, D.D. Haroske, T. Kühn and T. Ullrich, Mini-workshop: modern applications of s-numbers and operator ideals, Mathematisches Forschungs Institute Oberwolfach, Germany, 369-397, 8-14 February 2015.

[4] N. Dunford and J.T. Schwartz, Linear Operators I, Interscience Publishers, 1958.

[5] I.C. Gohberg and M.G. Krein, Introduction to the Theory of Linear Non-Selfadjoint Operators in Hilbert Space, American Mathematical Society, 1969.

[6] Z.I. Ismailov, Compact inverses of first-order normal differential operators, J. Math. Anal. Appl. 320, 266-278, 2006.

[7] Z.I. Ismailov, Multipoint normal differential operators for first order, Opuscula Math. 29, 399-414, 2009.

[8] Z.I. Ismailov, E. Otkun Çevik and E. Unluyol, Compact inverses of multipoint normal differential operators for first order, Electron. J. Differential Equations 89, 1-11, 2011.

[9] Z.I. Ismailov and E. Unluyol, Hyponormal differential operators with discrete spectrum, Opuscula Math. 30, 79-94, 2010.

[10] M.T. Karaev, M. Gürdal and U. Yamancı, Special operator classes and their properties, Banach J. Math. Anal. 7 (2), 74-88, 2013.

[11] M.A. Naimark and S.V. Fomin, Continuous direct sums of Hilbert spaces and some of their applications, Uspehi Mat. Nauk 10, 111-142, 1955, (in Russian).

[12] E. Otkun Çevik and Z.I. Ismailov, Spectrum of the direct sum of operators, Electron. J. Differential Equations 210, 1-8, 2012.

[13] A. Pietsch, Operators Ideals, North-Holland Publishing Company, 1980.

[14] A. Pietsch, Eigenvalues and s-Numbers, Cambridge University Press, 1987.

[15] R. Schatten and J. von Neumann, The cross-space of linear transformations, Ann. of Math. 47, 608-630, 1946.

[16] E. Schmidt, Zur theorie der linearen und nichtlinearen integralgleichungen, Math. Ann. 64, 433-476, 1907.

[17] H. Triebel, Über die verteilung der approximationszahlen kompakter operatoren in Sobolev-Besov-Raumen, Invent. Math. 4, 275-293, 1967. 